Cancer Cell. 2009;15(1):21-34.

11. Shojaei $F$, et al. Tumor refractoriness to anti-VEGF treatment is mediated by CD $11 \mathrm{~b}+\mathrm{Gr} 1+$ myeloid cells. Nat Biotechnol. 2007;25(8):911-920.

12. Shojaei F, et al. G-CSF-initiated myeloid cell mobilization and angiogenesis mediate tumor refractoriness to anti-VEGF therapy in mouse models. Proc Natl Acad Sci U S A. 2009;106(16):6742-6747.

13. Cascone $T$, et al. Upregulated stromal EGFR and vascular remodeling in mouse xenograft models of angiogenesis inhibitor-resistant human lung adenocarcinoma. J Clin Invest. 2011;121(4):1313-1328.

14. Bergers G, Song S, Meyer-Morse N, Bergsland E, Hanahan D. Benefits of targeting both pericytes and endothelial cells in the tumor vasculature with kinase inhibitors. J Clin Invest. 2003;111(9):1287-1295.

15. Helfrich I, et al. Resistance to antiangiogenic ther- apy is directed by vascular phenotype, vessel stabilization, and maturation in malignant melanoma. J Exp Med. 2010;207(3):491-503.

16. Nisancioglu MH, Betsholtz C, Genove G. The absence of pericytes does not increase the sensitivity of tumor vasculature to vascular endothelial growth factor-A blockade. Cancer Res. 2010;70(12):5109-5115.

17. Sennino B, et al. Cellular source and amount of vascular endothelial growth factor and platelet-derived growth factor in tumors determine response to angiogenesis inhibitors. Cancer Res. 2009; 69(10):4527-4536.

18. Lu C, et al. Targeting pericytes with a PDGF-B aptamer in human ovarian carcinoma models. Cancer Biol Ther. 2010;9(3):176-182.

19. Nolan-Stevaux O, et al. Differential contribution to neuroendocrine tumorigenesis of parallel Egfr signaling in cancer cells and pericytes. Genes Cancer. 2010;1(2):125-141.

20. Miller VA, O'Connor P, Soh C, Kabbinavar F. A randomized, double-blind, placebo-controlled, phase IIIb trial (ATLAS) comparing bevacizumab (B) therapy with or without erlotinib (E) after completion of chemotherapy with B for first-line treatment of locally advanced recurrent, or metastatic non-small cell lung cancer (NSCLC). J Clin Oncol. 2009;27(suppl):S18.

21. Kabbinavar FF, et al. Overall survival (OS) in ATLAS, a phase IIIb trial comparing bevacizumab (B) therapy with or without erlotinib (E) after completion of chemotherapy (chemo) with B for first-line treatment of locally advanced, recurrent, or metastatic non-small cell lung cancer (NSCLC). JClin Oncol. 2010;28(suppl):Abstract 7526.

\title{
Tolerogenic pDCs: spotlight on Foxo3
}

\section{Vincenzo Bronte}

Verona University Hospital and Department of Pathology, Immunology Section, University of Verona, Verona, Italy.

\begin{abstract}
Cancer creates a peculiar inflammatory environment enriched for transcription factors with a negative influence on adaptive immunity. In this issue of the JCI, Watkins and colleagues identify Foxo3 as a master regulator of the tolerogenic program in tumor-associated, plasmacytoid DCs (pDCs). Foxo3 enables pDCs to induce tolerance in tumor antigen-specific $\mathrm{CD8}^{+} \mathrm{T}$ cells, turning them into regulatory lymphocytes capable of inhibiting nearby $\mathrm{CD8}^{+} \mathrm{T}$ lymphocytes. Provision of tumor-specific $\mathrm{CD}^{+}$ $T$ helper cells interrupts this circuit by inhibiting Foxo3 expression and fully licensing the antigen-presenting ability of pDCs. These data identify a new target for therapeutic intervention and provide insight into the transcription factor interplay in myeloid cells recruited to the cancer microenvironment.
\end{abstract}

Complex responses to inflammatory stimuli require a sophisticated and coordinated transcriptional regulation activated by dedicated transcription factors (TFs) both at the single cell and tissue level. After LPS stimulation, a classical stimulus of the innate immune system, macrophages activate at least three classes of TFs: class I TFs that are shared by various cells and activated by signal-regulated, posttranslational modification; class II TFs that are synthesized de novo after stimulation; and class III TFs that are induced during cell differentiation and control complex phenotypic and functional changes (1). Studies in cancer are unveiling a network of negative transcriptional regulators of adaptive immunity in myeloid cells, which includes various members potentially belonging to the class III family.

Conflict of interest: The author has declared that no conflict of interest exists.

Citation for this article: J Clin Invest. doi:10.1172/ JCI57190.
The work from Watkins and colleagues reported in this issue of JCI identifies forkhead box O3 (Foxo3) as the main regulator of the immunosuppressive program in tumor-associated DCs (TADCs), which are mainly plasmacytoid DCs (pDCs), infiltrating prostate cancers (2). Human $\mathrm{CD} 123^{+} / \mathrm{CD} 304^{+} / \mathrm{CD} 11 \mathrm{c}^{-}$and mouse

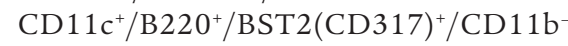
pDCs were isolated from either human prostate cancer specimens or cancers arising in transgenic adenocarcinoma of the mouse prostate (TRAMP) mice. Tumorconditioned pDCs but not pDCs isolated from other anatomical districts of tumorfree mice (including normal prostate) induced unresponsiveness to second stimulation with the antigen - that is, bona fide tolerance - in $\mathrm{CD}^{+} \mathrm{T}$ cells. Tolerance was antigen-specific and "infectious," since the $\mathrm{pDC}$-tolerized CD8 ${ }^{+} \mathrm{T}$ cells prevented naive $T$ cell proliferation in an antigen-unspecific manner, acting like Treg lymphocytes (Figure 1). Although pDCs from mouse tumor-draining lymph nodes were previously shown to directly activate mature $\mathrm{CD}^{+}$Tregs and prevent their conversion to $T$ helper 17 cells through a pathway requiring the activity of the enzyme indoleamine 2,3-dioxygenase (IDO) $(3,4)$, this work shows for the first time a direct conversion of $\mathrm{CD}^{+} \mathrm{T}$ cells toward a regulatory program.

\section{TADCs convert CD8+ $\mathrm{T}$ cells}

Watkins et al. found that TADC depletion by injection of anti-CD317 antibody resulted in increased activation and reduced Treg function of adoptively transferred $\mathrm{CD}^{+} \mathrm{T}$ cells reaching tumor mass: these cytotoxic $\mathrm{T}$ cells were specific for the main tumor antigen, the oncogene SV40, which drives tumorigenesis in TRAMP mice. TADC elimination also resulted in therapeutic benefit, since total urogenital tract and prostate weights, both indicators of tumor burden, were reduced in TADC-depleted TRAMP mice compared with those in control mice. Surprisingly, two amino acid metabolizing enzymes were upregulated in the TADCs: IDO and arginase 1 (ARG1). The authors used chemical inhibitors to provide evidence that these enzymes might be involved in the suppressive activity of TADCs on $\mathrm{CD}^{+} \mathrm{T}$ cells. However, only IDO inhibitors were effective in vitro, whereas both IDO and ARG inhibitors temporarily restored immune reactivity in vivo, indicating that ARG inhibitors might target this enzyme in other tumor-infiltrating cells, such as macrophages or subsets of classic CD $11 b^{+} / \mathrm{CD} 11 \mathrm{c}^{\text {hi }} / \mathrm{MHC} \mathrm{II}^{+}$ DCs, whose ARG1 overexpression was 
A
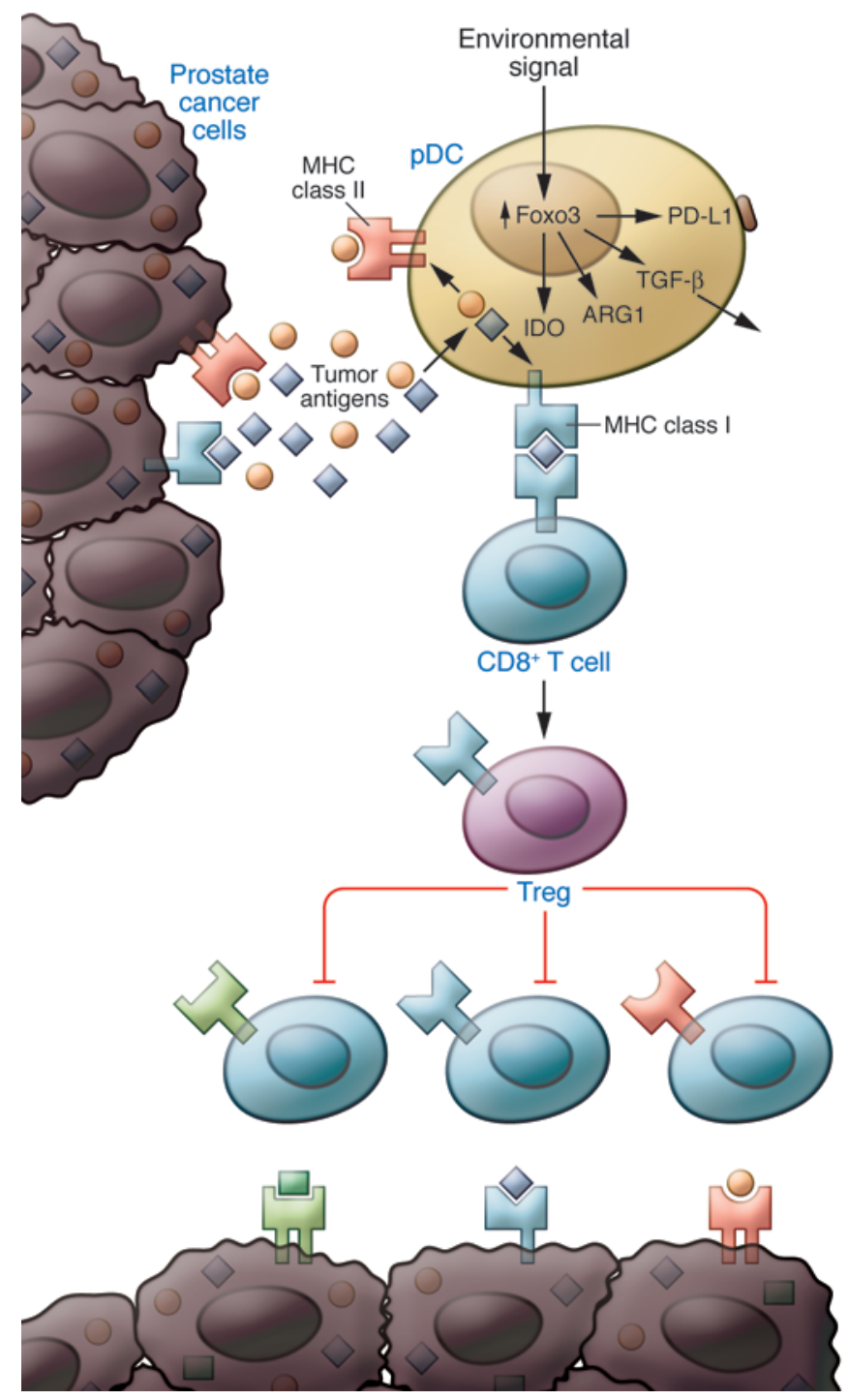

B

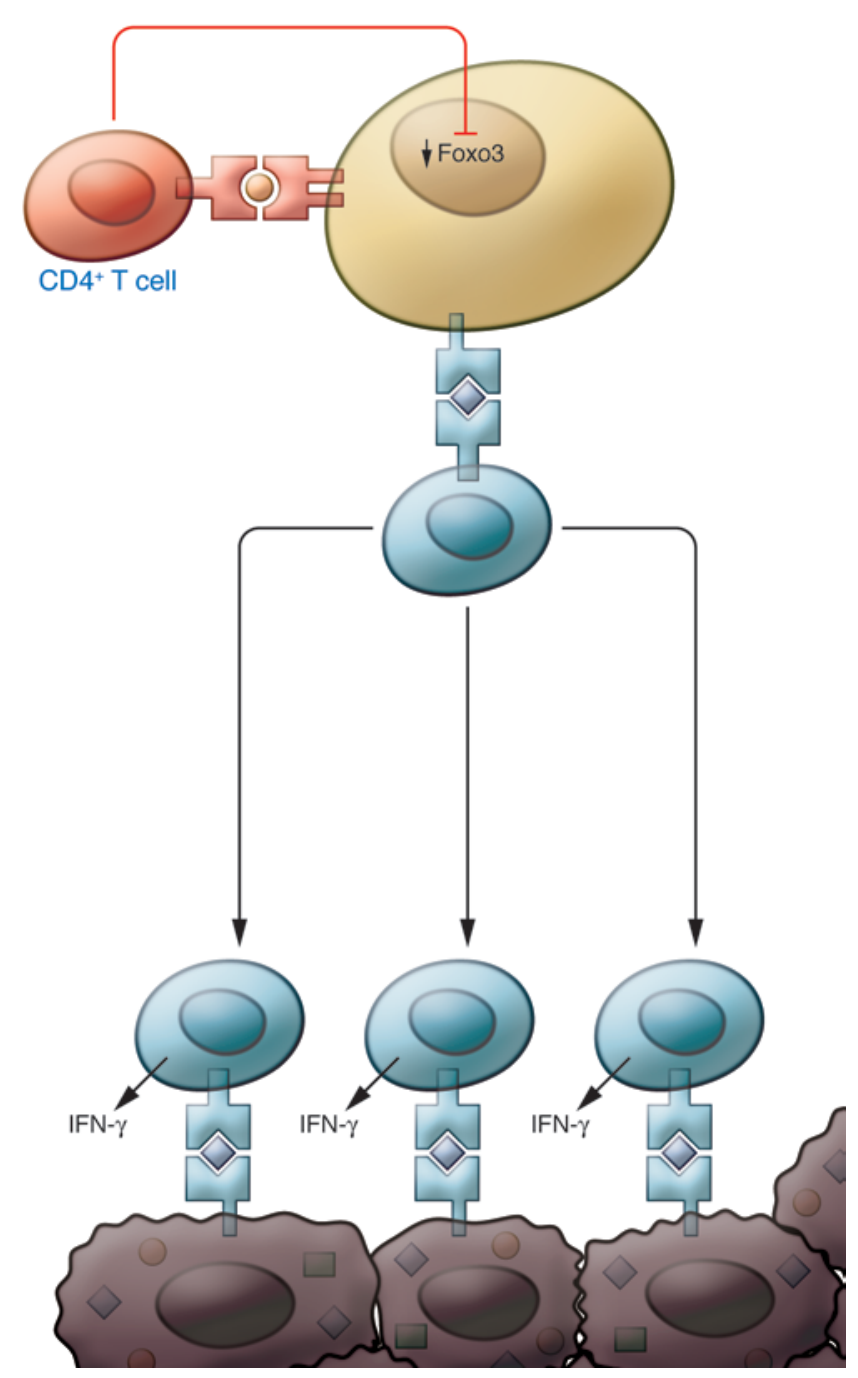

Figure 1

Interplay among leukocytes infiltrating prostate cancers. (A) pDCs enter the tumor environment, in which they can capture and present tumor antigens through class I (MHC I) and class II (MHC II) major histocompatibility molecules to CD8 ${ }^{+}$and CD4+ $\mathrm{T}$ cells specific for the antigen. Environmental signals drive the expression of Foxo3 that in turn controls the de novo synthesis of the amino acid-metabolizing enzymes IDO and ARG1, the cytokine TGF- $\beta$, and the surface inhibitory molecule PD-L1. After interaction with CD8+ T cells, Foxo3-expressing pDCs enforce conversion of $\mathrm{CD}^{+} \mathrm{T}$ cells into Tregs, which then block proliferation and effector functions on nearby $\mathrm{CD} 8^{+} \mathrm{T}$ lymphocytes, recognizing either the same or other tumor antigens. (B) This immunosuppressive network is disrupted by the generation of tumor-antigen specific CD4+ $4^{+}$cells that engage pDCs, turn off $\mathrm{FoxO}$, and make pDCs capable of fully stimulating CD8 ${ }^{+} \mathrm{T}$ cells, allowing their proliferation and acquisition of effector function, as highlighted by the recovered ability to release IFN- $\gamma$ and recognize cancer cells.

previously documented (5-7). The control exerted by IDO was transient since $\mathrm{T}$ cell suppression was reestablished within the tumor even in the presence of continuous IDO inhibitor.

Interestingly, other inhibitory pathways, such as TGF- $\beta$ and the programmed death $1 /$ programmed death ligand 1 (PD-1/PD-L1) axis, were activated in TADCs. Blocking either one was sufficient to ameliorate the tumor-induced unresponsiveness and increase the frequency and function of tumor-infiltrating $\mathrm{CD}^{+} \mathrm{T}$ lymphocytes, but no clear additive effect was observed and immune restoration was always transient. Therefore, inhibitory pathways appear to be redundant and temporally restricted, being replaced over time by alternative circuits, possibly in other tumorassociated cells (see below).

\section{Foxo3 promotes tolerogenic activity in TADCs}

By comparing data obtained either with siRNA-based silencing or genetic deletion, Watkins and colleagues convincingly demonstrated that Foxo3 expression in mouse and human TADCs represented a key factor for their tolerogenic activity not only in prostate cancer but also in other tumor models, including a mela- 


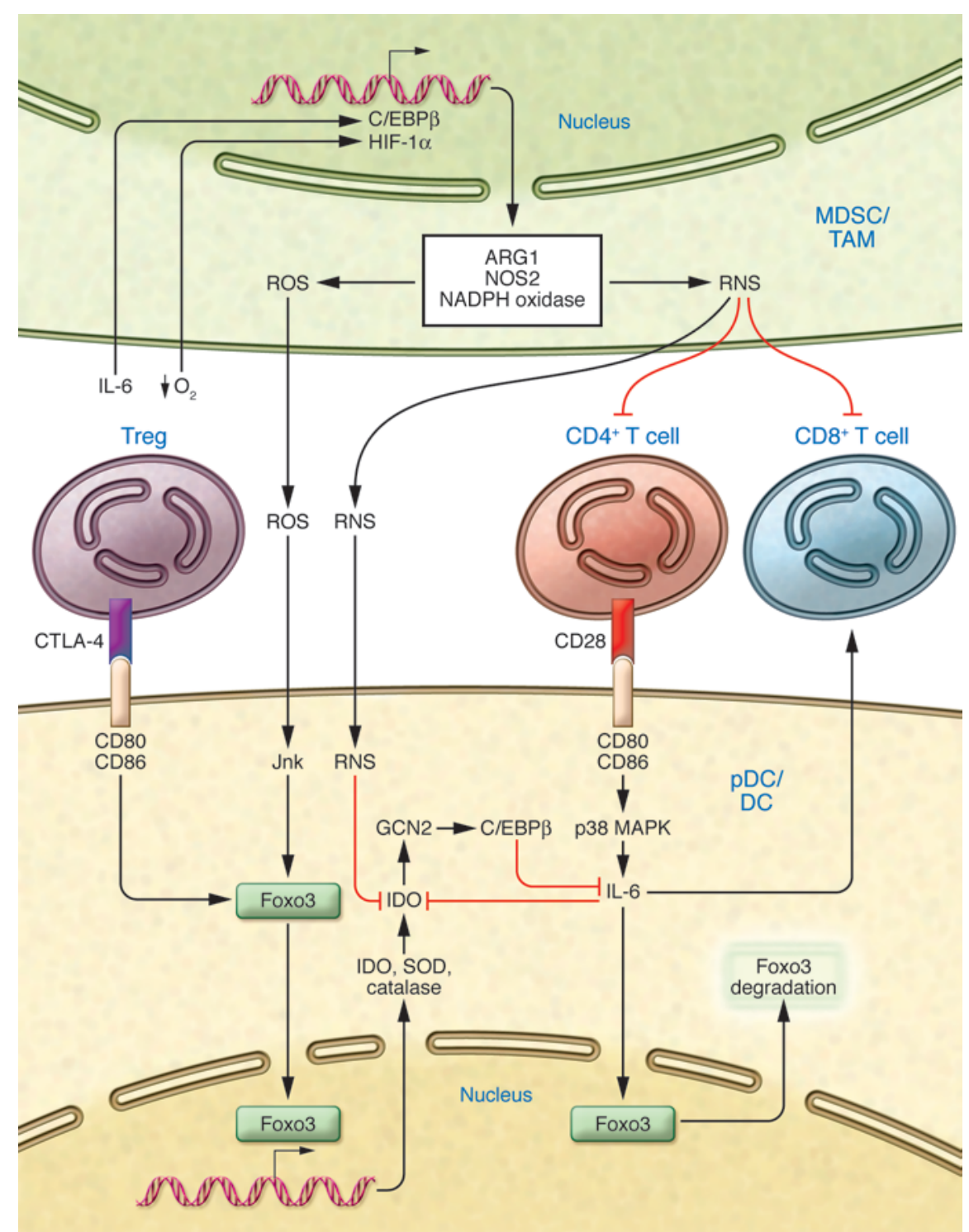

Figure 2

Immunosuppressive pathways and TFs in a changing cancer environment. In initial phases of cancer formation, Foxo3 regulation in pDCs and DCs depends on binding of the costimulatory molecules CD80 and CD86 by their ligands. CTLA-4 induces nuclear localization of Foxo3 and de novo transcription of the enzymes IDO, SOD, and catalase. Foxo3 nuclear export and subsequent degradation is stimulated by the binding of CD28, activation of p38 MAPK, and IL-6 production. A mini-circuit of negative cross-regulation is indicated, since IDO negatively regulates IL-6 production by depletion of L-tryptophan, activation of GCN2, and induction of the inhibitory isoform of C/EBP $\beta$. Through Jnk activation, environmentally produced ROS can also cause nuclear retention and activation of Foxo3. IL-6 is initially beneficial for the proliferation of tumor-specific CD8 ${ }^{+} \mathrm{T}$ lymphocytes; however, increased levels of IL-6 within tumor activate, via C/EBP $\beta$, the transcription of ARG1, NOS2, and NADPH oxidase in MDSCs and TAMs, leading to enhanced generation of ROS and RNS. An additive and possibly synergistic enhancement of this circuit comes from the activity of HIF-1 $\alpha$ in hypoxic areas of the tumor. RNS can turn off the immunosuppressive activity in $\mathrm{pDC}$ s and DCs by nitrating IDO but also directly inhibit $\mathrm{CD} 4^{+}$and $\mathrm{CD} 8^{+} \mathrm{T}$ lymphocytes, assuring the persistence of the negative influence on tumor-specific T cells.

noma. Foxo3 silencing correlated with an increased expression of the costimulatory molecule CD80 and the cytokine IL- 6 and a decrease in TGF- $\beta$, ARG1, and IDO. Block- ade of Foxo3-controlled pathways could be achieved in vitro by providing the proper immune "help signal" in the form of tumor antigen-specific $\mathrm{CD}^{+}$effector $\mathrm{T}$ cells:
pDCs cocultured with activated $\mathrm{CD}^{+} \mathrm{T}$ cells recognizing SV40 antigen had reduced levels of Foxo3 and were no longer able to induce tolerance in $\mathrm{CD}^{+} \mathrm{T}$ cells (Figure 1). Although the in vivo adoptive transfer of these $\mathrm{CD}^{+} \mathrm{T}$ cells led to a reduction in tolerogenic activity of TADCs, Foxo3 levels did not return to wild-type levels, and residual suppressive activity was detectable.

The $\mathrm{O}$ class members of the forkhead transcription superfamily are well conserved and have a wide range of impacts on cell function. Broadly, FOXO1, FOXO3, and FOXO4 are activated by metabolic signaling and are implicated in processes as diverse as cell death, longevity, and maintenance of the hematopoietic stem cell pool (reviewed in refs. 8 and 9). Growth factors such as insulin and insulin-like growth factor activate the phosphatidylinositol-3-OH (PI3) and Akt kinases, which phosphorylate FOXO proteins, resulting in nuclear exit and degradation $(8,9)$. Nuclear localization and transcriptional activation of FOXO proteins is induced after exposure to stress signals that control the mitogen-activated c-Jun N-terminal kinase (Jnk) $(8,9)$.

Foxo3 joins the STAT family, HIF-1 $\alpha$, and $\mathrm{C} / \mathrm{EBP} \beta$ in the list of TFs in the tumor environment driving the immunosuppressive programs in myeloid cells infiltrating either the tumor or the draining lymph nodes $(10,11)$. Despite their diversity, there is a common trend in these TFs regulating immunosuppression: they control the expression of enzymes involved in the metabolism of L-tryptophan and L-arginine, such as IDO, ARG, and NOS (reviewed in ref. 12).

\section{Activating Foxo3}

It is not clear what activates Foxo3 in TADCs, but some hints may be found in the literature. The most intensely investigated pathways of Foxo3 activation in DCs and pDCs involve the binding of CTL-A4 and CD28, mainly present on the surface of $\mathrm{CD}^{+}$Treg lymphocytes and activated CD4 ${ }^{+}$ T lymphocytes, respectively, to the costimulatory molecules CD80 and CD86, which has been described as "reverse signaling" (Figure 2). By interacting with costimulatory molecules, soluble CTLA-4 was found to induce nuclear localization of Foxo3 and trigger IDO expression by a pathway requiring induction of superoxide dismutase and blockade of peroxynitrite formation $(13,14)$. Conversely, binding of CD28 to either CD80 or CD86 inhibited IDO, and hence the immunosuppressive program in DCs, by activating p38 MAPK, SOCS3, and IL-6 
production (15). DCs from Foxo3-deficient mice are not tolerogenic but rather enhance $\mathrm{T}$ cell proliferation and survival by producing more IL-6, which is necessary for the expansion of antigen-activated $\mathrm{CD}^{+} \mathrm{T}$ lymphocytes (14). Interestingly, IL-6 and IDO seem to have an antithetic regulation: in pDCs, IDO enzymatic activation causes L-tryptophan starvation, consequent activation of the amino acid sensor general control nonrepressed 2 (GCN2) protein kinase, downstream activation of the inhibitory isoform of the TF C/EBP $\beta$, and repression of IL-6 production (3) (Figure 2).

Thus, it is possible to speculate that the Foxo3-driven program is a consequence of the intratumoral interaction between TADCs and CD4 ${ }^{+}$Tregs, known to occur in cancers of TRAMP mice (16), whereas the arrival of activated $\mathrm{CD}^{+} \mathrm{T}$ cells might turn off Foxo3 by supplying CD28-dependent signals (Figures 1 and 2). However, the presence of activated Foxo3 in tumor-associated $\mathrm{Gr}-1^{+}$cells and the induction of Foxo3 in $\mathrm{pDCs}$ exposed to tumor supernatant (A.A. Hurwitz and colleagues, unpublished observations) suggest that a more generalized induction of the TF might be occurring. Production of reactive nitrogen and oxygen species (RNS and ROS, respectively) is the most likely candidate for the intratumoral activation of Foxo3. As mentioned above, Foxo3 is maintained in the cytoplasm by the PI3K/Akt signaling pathways, and in the absence of signaling through Akt or in response to ROS, Foxo3 enters the nucleus and upregulates genes involved in oxidant metabolism (such as SOD), cell cycle arrest, and survival $(8,9)$. Prostate cancer offers a unique environment, rich in ROS and RNS, particularly peroxynitrites, deriving both from the neoplastic cells and from tumor-associated stromal cells (17). Nitrotyrosine content, an adduct product of the reaction between peroxynitrite and the amino acid L-tyrosine, is high in mouse and human prostate cancers and inversely correlates with the reactivity of tumor-infiltrating lymphocytes (17). Although it is not clear how this might occur, IDO itself has been suggested as a potential regulator of oxidative stress, since decreased protein nitration was detected in macrophages overexpressing IDO (18). Foxo3 might thus be part of a specific adaptive response to oxidative stress that pDCs, and other cells, encounter in tumor environment.

\section{Implications for a role of Foxo3 in promoting tolerance}

One might speculate that during initial tumorigenesis in TRAMP mice, reactive species trigger Foxo 3 expression in TADCs, but later the increased production results in a blockade of IDO (19), leading to IL-6 production. The suppressive mechanism might thus depend initially on TADCs and shift later to other tumor-infiltrating cells, such as myeloid-derived suppressor cells (MDSCs) and tumor-associated macrophages (TAMs). This scenario would be consistent with the limited efficacy of therapeutic approaches emerging from Watkins' article. By activating C/EBP $\beta$ TF, IL- 6 derived from different sources within the tumor microenvironment regulates the expression of ARG1, NOS2, and NADPH phagocytic oxidase (20), all of which contribute to increased ROS and RNS generation (Figure 2). Moreover, hypoxia can further sustain ARG1 and NOS 2 activity through HIF- $1 \alpha$ activation (5). Figure 2 summarizes our current knowledge about the interplay of some TFs controlling the tolerogenic environment in cancer. It remains to be determined whether and how TFs can cooperate, in the same or different cell types, a reasonable possibility suggested by the existence of known molecular interactions among FOXO3, STATs, and $\mathrm{C} / \operatorname{EBP} \beta$ (9).

\section{Acknowledgments}

I would like to thank Susanna Mandruzzato for the critical reading of the manuscript. This work was supported by grants from the Italian Ministry of Health, Fondazione Cassa di Risparmio di Padova e Rovigo, Italian Association for Cancer Research (AIRC), Association for International Cancer Research (AICR; grant 08-0518), and Istituto Superiore Sanità -Alleanza Contro il Cancro (project no. ACC8).

Address correspondence to: Vincenzo Bronte, Verona University Hospital and Department of Pathology, Immunology Section, Piazzale L.A. Scuro 10, 37134, Verona, Italy. Phone: 0039.045.8124007; Fax: 0039.045.8126455; E-mail: vincenzo. bronte@univr.it.
1. Medzhitov R, Horng T. Transcriptional control of the inflammatory response. Nat Rev Immunol. 2009; 9(10):692-703.

2. Watkins SK, et al. FOXO3 programs tumor-associated DCs to become tolerogenic in human and murine prostate cancer. J Clin Invest. 2011; 121(4):1361-1372.

3. Baban B, et al. IDO activates regulatory $\mathrm{T}$ cells and blocks their conversion into Th17-like T cells. J Immunol. 2009;183(4):2475-2483.

4. Sharma MD, et al. Plasmacytoid dendritic cells from mouse tumor-draining lymph nodes directly activate mature Tregs via indoleamine 2,3-dioxygenase. J Clin Invest. 2007;117(9):2570-2582.

5. Corzo CA, et al. HIF-1alpha regulates function and differentiation of myeloid-derived suppressor cells in the tumor microenvironment. J Exp Med. 2010;207(11):2439-2453.

6. Norian LA, et al. Tumor-infiltrating regulatory dendritic cells inhibit CD8+ T cell function via L-arginine metabolism. Cancer Res. 2009; 69(7):3086-3094.

7. Gallina $G$, et al. Tumors induce a subset of inflammatory monocytes with immunosuppressive activity on CD8+ T cells. J Clin Invest. 2006; 116(10):2777-2790.

8. Ouyang W, Li MO. Foxo: in command of T lymphocyte homeostasis and tolerance. Trends Immunol. 2011;32(1):26-33

9. van der Vos KE, Coffer PJ. FOXO-binding partners: it takes two to tango. Oncogene. 2008; 27(16):2289-2299.

10. Sonda N, Chioda M, Zilio S, Simonato F, Bronte V. Transcription factors in myeloid-derived suppressor cell generation [published online ahead of print January 10, 2011]. Curr Opin Immunol. doi:10.1016/ j.coi.2010.12.006

11. Condamine T, Gabrilovich DI. Molecular mechanisms regulating myeloid-derived suppressor cell differentiation and function. Trends Immunol. 2011; 32(1):19-25.

12. Grohmann U, Bronte V. Control of immune response by amino acid metabolism. Immunol Rev. 2010;236:243-264.

13. Fallarino F, et al. CTLA-4-Ig activates forkhead transcription factors and protects dendritic cells from oxidative stress in nonobese diabetic mice. J Exp Med. 2004;200(8):1051-1062.

14. Dejean AS, et al. Transcription factor FOXO3 controls the magnitude of $\mathrm{T}$ cell immune responses by modulating the function of dendritic cells. Nat Immunol. 2009;10(5):504-513.

15. Orabona C, et al. CD28 induces immunostimulatory signals in dendritic cells via CD80 and CD86. Nat Immunol. 2004;5(11):1134-1142.

16. Degl'Innocenti E, et al. Peripheral T-cell tolerance associated with prostate cancer is independent from CD4+CD25+ regulatory T cells. Cancer Res. 2008;68(1):292-300.

17. Bronte $\mathrm{V}$, et al. Boosting antitumor responses of $\mathrm{T}$ lymphocytes infiltrating human prostate cancers. JExp Med. 2005;201(8):1257-1268.

18. Keskin DB, Marshall B, Munn D, Mellor AL, Gearhart DA. Decreased protein nitration in macrophages that overexpress indoleamine 2, 3-dioxygenase. Cell Mol Biol Lett. 2007;12(1):82-102.

19. Fujigaki $\mathrm{H}$, et al. Nitration and inactivation of IDO by peroxynitrite. J Immunol. 2006; 176(1):372-379.

20. Marigo I, et al. Tumor-induced tolerance and immune suppression depend on the C/EBPbeta transcription factor. Immunity. 2010;32(6):790-802. 